\title{
The SsgA-like proteins in actinomycetes: small proteins up to a big task
}

\author{
Bjørn A. Traag · Gilles P. van Wezel
}

Received: 3 December 2007 / Accepted: 29 January 2008/Published online: 14 February 2008

(C) The Author(s) 2008

\begin{abstract}
Several unique protein families have been identified that play a role in the control of developmental cell division in streptomycetes. The SsgA-like proteins or SALPs, of which streptomycetes typically have at least five paralogues, control specific steps of sporulation-specific cell division in streptomycetes, affecting cell wall-related events such as septum localization and synthesis, thickening of the spore wall and autolytic spore separation. The expression level of SsgA, the best studied SALP, has a rather dramatic effect on septation and on hyphal morphology, which is not only of relevance for our understanding of (developmental) cell division but has also been succesfully applied in industrial fermentation, to improve growth and production of filamentous actinomycetes. Recent observations suggest that SsgB most likely is the archetypal SALP, with only SsgB orthologues occurring in all morphologically complex actinomycetes. Here we review 10 years of research on the SsgA-like proteins in actinomycetes and discuss the most interesting regulatory, functional,
\end{abstract}

Electronic supplementary material The online version of this article (doi:10.1007/s10482-008-9225-3) contains supplementary material, which is available to authorized users.

B. A. Traag · G. P. van Wezel $(\square)$

Microbial Development, Leiden Institute of Chemistry, Leiden University, P.O. Box 9502, 2300 RA Leiden,

The Netherlands

e-mail: g.wezel@chem.leidenuniv.nl phylogenetic and applied aspects of this relatively unknown protein family.

Keywords Streptomyces - SALP · Phylogeny · Peptidoglycan · Cell division · Development

\section{Introduction}

Actinomycetes have an unusually complex life cycle, many aspects of which are globally similar to those observed in some lower eukaryotes, which makes them particularly interesting for the study of bacterial development and evolution (Chater and Losick 1997). One of the best characterized genera among the actinomycetes is Streptomyces. As producers of over half of the known antibiotics, the Gram-positive soil-dwelling filamentous streptomycetes are a paradigm of secondary metaboliteproducing microorganisms (Chater and Losick 1997; Hopwood 1999), with Streptomyces coelicolor A3(2) as the most-studied streptomycete (Hopwood 1999). Development of streptomycetes is initiated by the germination of a spore, from which typically two hyphae are produced, which continue to grow and branch to form a vegetative mycelium. Exponential growth is achieved by apical (tip) growth and branching (Flärdh 2003), with a complex mycelial network as the result. The vegetative hyphae consist of syncytial cells separated by occasional cross-walls, laid down at 5-10 $\mu \mathrm{m}$ intervals (Wildermuth and Hopwood 1970). When development is initiated an aerial mycelium is produced, with hydrophobic hyphae breaking through 
the moist surface, erected into the air. This is the start of the reproductive phase, initiated in response to nutrient depletion and the resulting requirement of mobilization. Eventually, sporulation-programmed hyphae are formed in a process requiring a complex, spatial and temporal genetic programming scheme that is switched on upon nutrient limitation (Chater 1998). During sporulation long chains of unigenomic spores are formed from multigenomic aerial hyphal compartments.

Multiple cell division during sporulation of streptomycetes requires an unparalleled complex coordination of septum-site localization, cell division and DNA segregation and coordination (Flärdh et al. 2000; McCormick et al. 1994; Schwedock et al. 1997; Wildermuth and Hopwood 1970). Penicillinbinding proteins (PBPs) are key enzymes for the synthesis of the bacterial peptidoglycan, both during growth and during cytokinesis (Errington et al. 2003; Holtje 1998; Stewart 2005). The best-studied PBPs are PBP2, which is required specifically for lateral cell-wall synthesis in E. coli (Den Blaauwen et al. 2003), and FtsI (PBP3), which is part of the divisome and essential for synthesis of the septal peptidoglycan (Botta and Park 1981). Streptomycetes contain many PBPs and a number of these are developmentally controlled, suggesting a role specifically during sporulation (Hao and Kendrick 1998; Noens et al. 2005). During maturation, spores are separated in a process that most likely resembles the separation of mother and daughter cells during cell division of unicellular bacteria, involving several autolytic enzymes such as amidases, lytic transglycosylases and endopeptidases (Heidrich et al. 2002). While the cell division machinery of streptomycetes strongly resembles that of other bacteria (Flärdh and van Wezel 2003), the control of septum formation is very different in these organisms. Streptomycetes lack the MinC, MinE and SulA proteins that control septumsite localization (Autret and Errington 2001; Marston et al. 1998), the nucleoid occlusion system (NOC) that coordinates septum synthesis and DNA segregation (Wu and Errington 2004), as well as some crucial Z-ring anchoring proteins such as FtsA and ZipA (Errington et al. 2003; Lowe et al. 2004). How cell division is controlled in streptomycetes is unclear. In sporulation-committed aerial hyphae FtsZ organizes into spiral-shaped intermediates along the length of the aerial hyphal cell, eventually forming up to a hundred Z-rings per aerial hyphae (Grantcharova et al. 2005). At this stage MreB localizes to the septa, suggesting this actin-like cytoskeletal protein may assist in cell division (Mazza et al. 2006).

Instead of the canonical cell division control proteins, several unique protein families have been identified that play a role in the control of cell division in streptomycetes, and notably the CrgA-like proteins and the SsgA-like proteins (Chater and Chandra 2006; Flärdh and van Wezel 2003). CrgA-like proteins comprise a family of small integral membrane proteins, thought to play a role in the inhibition of FtsZring formation during Streptomyces development (Del Sol et al. 2006). In this review, we will have a closer look at the SsgA-like proteins or SALPs, which play an important role in the control of sporulation-specific cell division in sporulating actinomycetes.

\section{The cell division activator SsgA}

In the mid-90s Kawamoto and Ensign identified a genomic DNA fragment of Streptomyces griseus that inhibited submerged sporulation of a hyper-sporulating S. griseus strain at multiple copies (Kawamoto and Ensign 1995). The same genomic fragment induced fragmented growth of the otherwise branching mycelial filaments and the responsible gene was designated ssgA (for sporulation of Streptomyces griseus). A direct correlation between SsgA accumulation and the onset of sporulation in wild-type cells, and the failure of some developmental mutants of S. griseus to accumulate SsgA, further demonstrated the sporulation-related function of SsgA (Kawamoto et al. 1997). SsgA is member of a family of proteins now known as the SsgA-like proteins (SALPs), which are small acidic 14-17 kDa proteins that do not carry any known protein motif (see next section). Remarkably, even to date there is not a single protein in the databases that has significant sequence similarity to hint at the possible mode of action for the SALPs.

ssgA null mutants of both $S$. coelicolor and $S$. griseus produce an aerial mycelium but fail to sporulate except on mannitol-containing media, where some spores are produced after prolonged incubation; this makes $s s g A$ a rather unique example of a conditional white (whi) mutant (Jiang and Kendrick 2000; van Wezel et al. 2000a). Later studies showed that SsgA directly activates sporulation-specific cell division, and over-production results 
in a dramatic morphological change of the vegetative hyphae of $S$. coelicolor; the vegetative hyphae become approximately twice as wide (average of $800 \mathrm{~nm}$ instead of $400-500 \mathrm{~nm}$ ) and form spore-like compartments separated by massive and aberrant septa, resulting in hyper-fragmenting hyphae in submerged culture that occasionally produce submerged spores (van Wezel et al. 2000a; van Wezel et al. 2000b). The large impact of SsgA on morphogenesis was underlined by microarray analysis, which showed that deletion of $\operatorname{ssg} A$ affects expression of an unprecedented large number of genes, with many hundreds of genes up- or downregulated by at least two-fold, including most developmental genes (e.g. bld, whi and ssg genes), as well as many genes involved in DNA segregation and topology (Noens et al. 2007). The remarkable upregulation of many of the known bld and whi genes, which are essential for aerial mycelium and spore formation, respectively, is best explained as a stress response to try and compensate for the absence of an important morphogen (i.c. ssgA). The same is probably true for the strong upregulation of ftsI (septum synthesis) and of $\operatorname{divIVA}$ (apical growth), whose functions relate to and may be assisted by SsgA. The genes that are by far the most strongly upregulated in $s s g A$ mutants are the chaplin and rodlin genes. These genes encode hydrophobic proteins that form the water-repellent sheath of aerial hyphae that allows them to break through the soil surface (Claessen et al. 2003; Claessen et al. 2004; Elliot et al. 2003). Further research is required to explain the correlation between these developmental coat proteins and SsgA.

\section{The SsgA-like proteins exclusively occur in morpologically complex actinomycetes}

SALPs typically are proteins of $120-150$ amino acids (aa) with a sequence identity between $30 \%$ and $50 \%$. Seven SALPs were found in the fully sequenced genomes of S. coelicolor (designated SsgA-G; in this review we will use the $S$. coelicolor nomenclature throughout, unless stated otherwise) and in S. scabies, six in $S$. avermitilis, and five in the recently sequenced genome of $S$. griseus. Alignment of the 24 known SALP proteins of streptomycetes (presented in Traag et al. 2007) showed that, despite the relatively low conservation, $11 \%$ (16 residues) of all amino acids are completely conserved. Surprisingly, this number does not change much when all 53 SALPs are taken into account, including the SALPs from sometimes quite distantly related actinomycetes. In that case 11 residues are still fully conserved, and if one of the SALPs from each of the Frankia species (FrS2 in Fig. 1) is left out of the comparison, 15 residues are completely conserved among 50 SALP sequences. A random mutant library was created for $S$. coelicolor $s s g A$, from which approximately $800 \mathrm{ssg} A$ variants were sequenced and screened for their ability to complement the $s s g A$ null mutant. The essential residues for SsgA function were predominantly found in the first two thirds of the analysed part of the protein and most of these are highly conserved among all SALPs, while the penalty for mutations in the C-terminal domain was much lower. Three aa residues (L39, D68, and S99) are essential for SsgA function but are not significantly conserved in other SALPs and these may therefore have an SsgA-specific function (Traag et al. 2007).

Five of the SALPs, namely SsgA, SsgB, SsgD, $\mathrm{SsgE}$ and $\mathrm{SsgG}$, have orthologues in all streptomycetes analysed (Noens et al. (2005) and our unpublished hybridization data), with the exception of SsgG, absent from the $S$. avermitilis genome. Additionally, a few species-specific SALPs are typically found in all streptomycetes. SALPs can be divided into phylogenetic subfamilies, namely the SsgA branch, the SsgBG branch, the SsgDE branch and the species-specific SALPs, which include SsgC and SsgF (Fig. 1). Unexpectedly, the genome of $S$. griseus contains an additional three much larger proteins with an approximately 120 aa C-terminal SsgA-like domain, which contains the typical sequence identity to other SALPs of approximately 30-50\%. Two of these (SGR7098t and SGR41t) are identical proteins of 654 aa, while the third (SGR128) is a 651 aa protein with an end-to end sequence identity of $67 \%$ to the other two (approximately $84 \%$ in the 120 aa SsgA-like domain). Apart from the SsgA-like domain the remainder of these proteins have no significant similarity to other proteins.

So far SALPs have exclusively been found in actinomycetes. The genomes of Thermobifida fusca, Kineococcus radiotolerans, Nocardioides, Acidothermus cellulolyticus, Salinispora tropica and Salinispora arenicola, all of which are either not known to form spores or produce single spores borne on the vegetative 
Fig. 1 Phylogenetic tree of the SALP proteins. Phylogenetic analysis of all SsgA-like proteins (SALPs) from different actinomycetes was performed using the CLUSTALX program (Thompson et al. 1997). Accession numbers and other information on the proteins in the tree are listed in Supplementary Table S1. The three subfamily branches (SsgA, SsgBG, and $\mathrm{SsgDE}$ ) are indicated

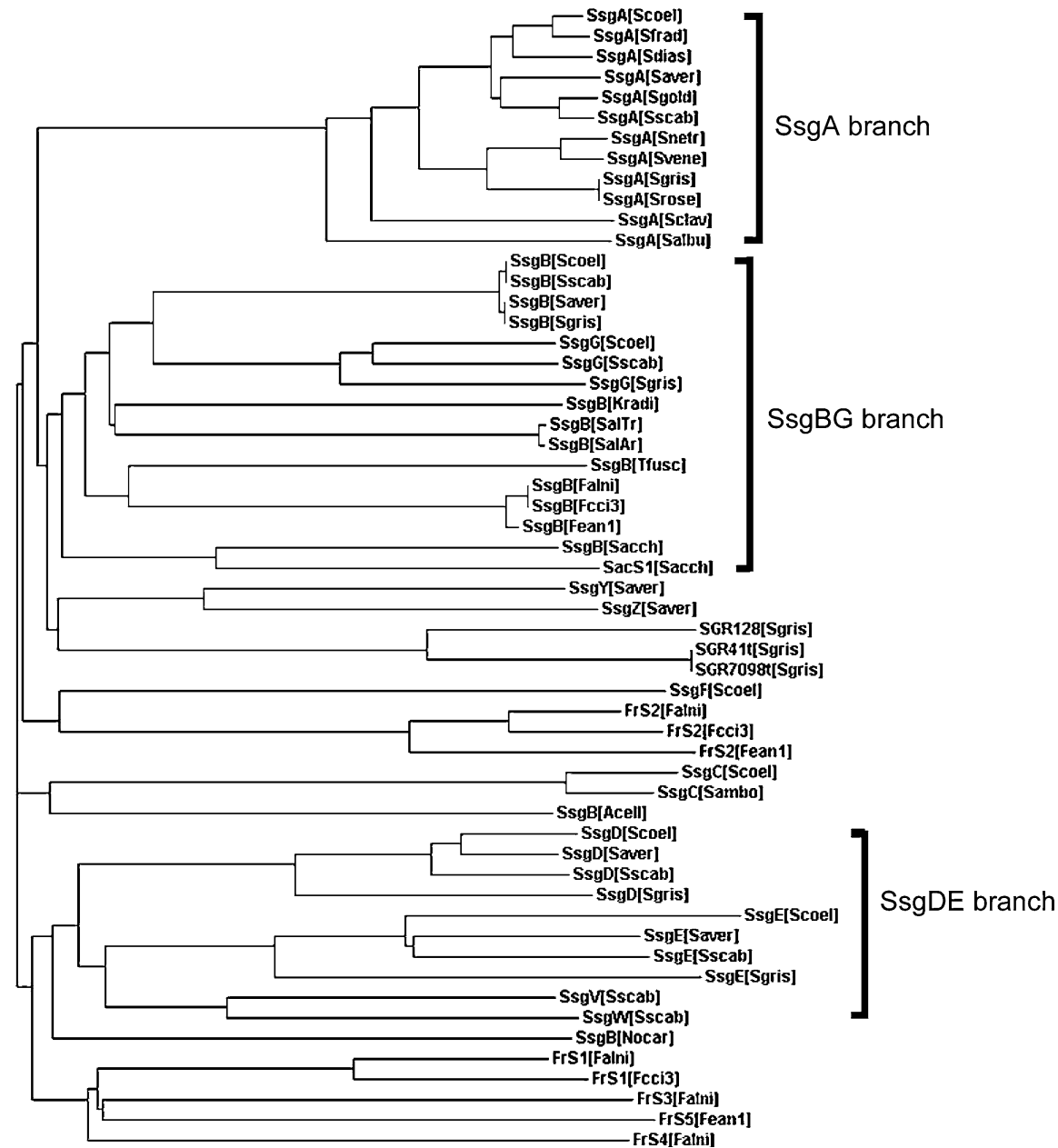

mycelium, contain a single ssg gene, which is most likely functionally related to $\operatorname{ss} B$ (see below). Similar to streptomycetes, the multispore-forming actinomycetes have multiple homologues, namely two in Saccharopolyspora erythraea, three in Frankia sp. CcI3 and Frankia sp. EAN1pec and five in Frankia alni (Table 1). Remarkably, there is a clear correlation between the complexity of the morphology of actinomycetes and the number of SALPs found in actinomycetes. No SALPs were identified in Mycobacterium, Rhodococcus or Corynebacterium species.

\section{SsgB is most likely the SALP archetype}

Analysis of the genetic locus of the single SALPencoding genes of Thermobifida, Kineococcus, Nocardioides, Acidothermus and Salinispora showed that all resembled the gene organization around $s \mathrm{sg} B$ in Streptomyces. In fact, all SALP-containing actinomycetes have one ssg gene with a similar genetic locus to Streptomyces ssgB (BAT and GPvW, unpublished data), which is preceded by a homologue of SCO1540 (the gene directly upstream of $S$. coelicolor $\operatorname{ss} B$ ) in most actinomycetes and in all genera a gene for tRNA ${ }^{\mathrm{val}}$ somewhat further upstream. A number of other genes are conserved in several genera, for example at least three additional tRNA genes are found in all except Salinispora, and a threonine-tRNA synthetase (SCO1531) is present in Streptomyces, Acidothermus, Kineococcus, Nocardioides and Thermobifida. Hence, gene syntheny evidence strongly suggests that all SALP-encoding genes have been derived from spread and/or gene duplication of $s \mathrm{sg} B$ in actinomycetes and that perhaps this gene has a universally conserved function in actinomycete 
Table 1 Distribution of SALPs across actinomycetes

\begin{tabular}{|c|c|c|c|c|}
\hline Strain & $\begin{array}{l}\text { Genome } \\
\text { accession }\end{array}$ & $\begin{array}{l}\text { Genome size } \\
(\mathrm{Mbp})\end{array}$ & $\begin{array}{l}\text { Number of } \\
\text { SALPs }\end{array}$ & $\begin{array}{l}\text { Cell morphology; } \\
\text { development }\end{array}$ \\
\hline Streptomyces avermitilis & BA000030 & 9 & 6 & \multirow{4}{*}{$\begin{array}{l}\text { Filamentous growth, spore chains } \\
\text { on aerial hyphae }\end{array}$} \\
\hline Streptomyces coelicolor & AL645882 & 8.7 & 7 & \\
\hline Streptomyces griseus & $\begin{array}{l}\text { (project ID: } \\
20085 \text { ) }\end{array}$ & 8.5 & 5 & \\
\hline Streptomyces scabies & $\begin{array}{l}\text { (project } \\
\text { ID:12985) }\end{array}$ & 10.1 & 7 & \\
\hline Frankia alni & CT573213 & 7.5 & 5 & \multirow{3}{*}{$\begin{array}{l}\text { Filamentous growth, multilocular sporangia } \\
\text { either terminally or intercalary }\end{array}$} \\
\hline Frankia sp. CcI3 & СР000249 & 5.4 & 3 & \\
\hline Frankia sp. EAN1pec & AAII00000000 & 9 & 3 & \\
\hline $\begin{array}{l}\text { Saccharopolyspora } \\
\quad \text { erythraea }\end{array}$ & AM420293 & 8.2 & 2 & $\begin{array}{l}\text { Filamentous growth, short spore chains } \\
\text { on fragmented aerial hyphae }\end{array}$ \\
\hline $\begin{array}{l}\text { Acidothermus } \\
\text { cellulolyticus }\end{array}$ & СР000481 & 2.4 & 1 & Slender floculles, non-spore forming \\
\hline $\begin{array}{l}\text { Kineococcus } \\
\quad \text { radiotolerans }\end{array}$ & AAEF00000000 & 4.9 & 1 & $\begin{array}{l}\text { Cocci with polar flagella or symmetrical multi-cell } \\
\text { clusters, non-spore forming. Ageing colonies form an } \\
\text { extracellular polymer shell around individual colonies }\end{array}$ \\
\hline Nocardioides sp. JS614 & СР000509 & 4.9 & 1 & Single rods/cocci, non-spore forming \\
\hline Salinispora arenicola & AAWA0000000 & 5.7 & 1 & \multirow{2}{*}{$\begin{array}{l}\text { Filamentous growth, single spores borne } \\
\text { on substrate mycelium }\end{array}$} \\
\hline Salinispora tropica & AATJ00000000 & 5.2 & 1 & \\
\hline Thermobifida fusca & CP000088 & 3.6 & 1 & $\begin{array}{l}\text { Filamentous growth, single spores borne } \\
\text { on dichotomously branched sporophores }\end{array}$ \\
\hline
\end{tabular}

morphogenesis. In a phylogenetic tree nearly all of the putative SsgB orthologues group together in the above mentioned SsgBG branch (Fig. 1), with the exception of the orthologues from the non-sporulating Acidothermus and Nocardioides. Whether these two SALP orthologues are still functional in these actinomycetes remains to be elucidated. More phylogenetic evidence for the importance of $\mathrm{SsgB}$ is provided by the fact that SsgB orthologues found in different species within a specific genus are almost completely conserved. This is true for the SsgBs from Streptomyces, from Frankia and from the salt-water actinomycete Salinispora. The orthologues from Streptomyces are identical except for aa position 150 (Gln or Thr), those from Salinispora differ only at aa position 137 (Asn or Ser), and in Frankia two orthologues are identical while the third has three aa changes (two conserved Ile/Val changes, and more importantly Ser or Ala at aa position 105). Furthermore, many nucleotide changes occur that do not lead to changes in the predicted proteins. This extraordinary conservation within genera perhaps reflects an inflexible co-evolution with an interaction partner. $\operatorname{ssg} B$ (and to a lesser extent $\operatorname{ssg} A$, which is not essential for sporulation on most mannitol-containing media) is one of the very few known whi genes that are essential for sporulation but do not encode a DNA binding protein. $s s g B$ mutants produce long aseptate aerial hyphae in seemingly 'immortal' white colonies (Keijser et al. 2003), indicating a possible role for $\mathrm{SsgB}$ in the cessation of aerial growth prior to the onset of sporulation-specific cell division. Interestingly, many electron-dense granules were seen in hyphae of both $\operatorname{ssg} B$ mutants and PBP2 mutants, which perhaps reflect the accumulation of peptidoglycan subunits in both mutants (Noens et al. 2005). Importantly, the $s s g B$ genes from Salinispora tropica and Saccharopolyspora erythraea restored sporulation to the otherwise non-sporulating $\operatorname{ss} B$ mutant of $S$. coelicolor, even though end-to-end sequence homology to $S$. coelicolor $\operatorname{ssg} B$ for both is only around $50 \%$ (BAT and GPvW, unpublished data). This strongly suggests that the SsgB proteins of Salinispora and Saccharopolyspora are indeed functional orthologues of SsgB. However, defects in septum placement, DNA segregation and spore size 
indicate that control of septum-site localization depends on (the amino acid sequence of) SsgB. Indeed, localization of $\mathrm{SsgB}$ to both tips of growing aerial hyphae and to immature sporulation septa suggests the protein functions in both tip growth and cell division (E.E. Noens, J. Willemse and GPvW, unpublished data).

Phylogenetically the closest relative of $\mathrm{SsgB}$ is SsgG (see Fig. 1), and this SALP plays a role specifically in the control of septum-site localization. ss $G$ mutants have a light grey phenotype, resulting from the production of significantly fewer spores than wild-type S. coelicolor. Closer inspection revealed that sporulation septa are regularly 'skipped', resulting in many spores of exactly two, three or even four times the normal size. This showed that SsgG is required to ensure that the divisome is localised to all division sites (Noens et al. 2005). Interestingly, despite the lack of cell division in the multiple-sized spores of the $\operatorname{ssg} G$ mutant the chromosomes were segregated normally, which clearly demonstrates that septum synthesis is not a prerequisite for DNA segregation in streptomycetes.

Streptomyces coelicolor ssgC-F mutants produced an abundance of grey-pigmented spores after a few days of incubation, and $\operatorname{ssg} C$ mutants even hypersporulate. Microscopic analysis showed that aerial hyphae of the $\operatorname{ss} C$ mutant produce very long ladders of septa, resulting in seemingly endless spore chains, while at the same time chromosome segregation in aerial hyphae was disturbed. In fact, $s s g C$ mutants in many ways resemble strains over-producing SsgA and vice versa, and it was therefore proposed that SsgC may function as an antagonist of SsgA (Noens et al. 2005). Orthologues of $s s g C$ are so far only found in streptomycetes that have a low expression of $\operatorname{ss} g A$ under conditions of normal growth and as a result produce large clumps in liquid-grown cultures, namely in $S$. coelicolor, in $S$. ambofaciens and, as determined by hybridization studies, in $S$. lividans (GPvW, unpublished). Mutation of $s s g D$ pleiotropically affected integrity of the cell wall in aerial hyphae and spores, with many spores lacking the typical thick peptidoglycan layer, which rather resembles the wall of aerial hyphae. Finally, correct autolytic spore separation depends on SsgE and SsgF. $s s g E$ mutants produce predominantly single spores indicating an enhanced or accelerated autolytic activity, while mutation of $s s g$ leads to incomplete detachment of spores, which remain attached by a thin peptidoglycan linkage, suggesting reduced or incorrect function of the secreted lytic transglycosylase SLT (Noens et al. 2005).

\section{Transcriptional regulation of the $s s g$ genes in streptomycetes}

Microarray analysis of $S$. coelicolor M145 and MT1110 and promoter-probe experiments in S. coelicolor indicated that all $s s g$ genes except $s s g D$ are developmentally regulated (Noens et al. 2005; Noens et al. 2007). In contrast to the other $s s g$ genes, $s s g D$ is transcribed at a much higher level and its transcription is life cycle-independent (Traag et al. 2004), suggesting a role for $\mathrm{SsgD}$ at different stages of the life cycle. Furthermore, all ssg genes but $s s g D$ are catabolically repressed by glucose. The transcription of $s s g A$ has been studied extensively (see below). The only other $s s g$ gene whose transcription has been studied in more detail is $s s g$. Transcription of $s s g B$ is directed from a single promoter $52 \mathrm{nt}$ upstream of the most likely ATG start codon, resulting in a protein of 137 aa. Expression occurs in a life-cycle dependent manner and is strongly activated towards sporulation (Kormanec and Sevcikova 2002). In an $E$. coli two-plasmid system the $s s g B$ promoter was found to be active in the presence of several members of the SigB-like sigma factor family (i.e. $\sigma^{\mathrm{B}}, \sigma^{\mathrm{F}}$ and $\sigma^{\mathrm{H}}$ ). SigB-like $\sigma$ factors, of which the $S$. coelicolor genome encodes nine paralogues, are implicated in morphological differentiation and/or responses to different stresses (Kelemen et al. 2001; Lee et al. 2004). Expression of $\operatorname{ssg} B$ was unaffected in an $\operatorname{sig} F$ mutant of $S$. coelicolor, while in a sigH mutant no $\operatorname{ss} B$ transcripts were detected. Furthermore, Histagged $\sigma^{\mathrm{H}}$ initiated transcription from the $\operatorname{sig} B$ promoter in in vitro run-off transcription assays (Kormanec and Sevcikova 2002). However, since a sigH mutant of $S$. coelicolor still produced some spores (Sevcikova et al. 2001) and a $\operatorname{ssg} B$ mutant does not, sig $H$ cannot be solely responsible for transcription of $\operatorname{ssg} B$.

\section{Transcriptional and translational control of $\operatorname{ssg} A$}

Transcription of $s s g A$ is strongly induced towards sporulation in both S. griseus and S. coelicolor (Traag 
et al. 2004; Yamazaki et al. 2003). In both strains transcription is directed from two transcriptional start sites, one of which is essentially the same in both species ( $\mathrm{p} 1$ in $S$. coelicolor and $\mathrm{p} 2$ in $S$. griseus; indicated as "B" in Fig. 2) while the other is different. Transcriptional analysis in six early whi mutants of S. coelicolor (whiA, whiB, whiG, whiH, whiI and whiJ) revealed that both $s s g A$ and $s s g R$ are transcribed independently from these crucial sporulation genes (Traag et al. 2004). Directly upstream of $s s g A$ lies a gene called $s s g R$, encoding an IclR-type DNA binding transcriptional regulator. In-frame deletion mutants of $s s g R$ in $S$. griseus and $S$. coelicolor resulted in a sporulation deficient phenotype very similar to that of the $s s g A$ mutants, and transcription of both $s s g A$ and $s s g R$ is strongly induced towards the onset of sporulation (Traag et al. 2004; Yamazaki et al. 2003), suggesting that ssgR regulated $s s g A$ transcription. Indeed, transcriptional and DNA binding studies showed that ssgA is transactivated by and completely dependent on SsgR in S. coelicolor (Traag et al. 2004). Suggestively, the A/T-rich region around the stop codon of $s s g R$ lies at the centre of a DNA fragment that was bound by SsgR in vitro and is strongly conserved among different streptomycetes, while the sequences surrounding it are far less well conserved (Fig. 2). The conserved region around the stop codon is quite possibly the binding site for SsgR (Traag et al. 2004). Comparison of the global expression profiles of the $s s g A$ and $s s g R$ mutants of $S$. coelicolor by microarray analysis revealed extraordinary similar expression profiles, providing further evidence that $s s g A$ may well be the only target of SsgR (Noens et al. 2007). In contrast, in $S$. griseus expression of $s s g A$ is only slightly affected by SsgR and instead fully dependent on AdpA, a transcriptional activator in the A-factor regulatory cascade essential for differentiation and antibiotic production of $S$. griseus (Horinouchi and Beppu 1994; Ohnishi et al. 2005). AdpA binds to three adjacent sites upstream of $s s g A$ (Yamazaki et al. 2003). Conversely, in $a d p A$ (bldH) mutants of $S$. coelicolor transcription of $s s g A$ was readily detected, though deregulated in earlier stages of the life cycle (Traag et al. 2004). Different functions for the SsgR orthologues from both species are also suggested by the observation that $s s g R$ from $S$. griseus failed to complement the $S$. coelicolor ssgR mutant (Traag et al. 2004). The discrepancy in the transcriptional control of $\operatorname{ssg} A$ and the resulting enhanced expression in S. griseus is at least one of the main reasons for the major morphological differences between $S$. coelicolor (clumps) and $S$. griseus (submerged spores) in submerged cultures.

There has long been a controversy about the position of the $\operatorname{ssg} A$ translational start site. While initially $S$. griseus $s s g A$ was considered to encode a 145 aa protein (Kawamoto and Ensign 1995), the presence of a more likely ribosome binding site (RBS) further downstream led to reassignment of the translational start to the third of three in-frame ATG codons, that lies 30 nucleotides (10 codons) downstream of the originally predicted start (Kawamoto et al. 1997). Alignment of ten ssgA ORFs and flanking regions obtained from the sequenced genomes of $S$. avermitilis, $S$. coelicolor, $S$. griseus, and $S$. scabies and from sequenced ssgA clones in our laboratory (namely from S. albus, S. clavuligerus, $S$. diastatochromogenes, $S$. fradiae, $S$. roseosporus, and $S$. venezuelae), showed that two alternative ATG start codons and the putative RBS sequences preceding them are fully conserved (Fig. 2), with precious few differences in the sequences between these possible start sites among the different $s s g A$ orthologues. This suggests that perhaps both ATG codons could function as start codons. Indeed, we recently obtained evidence from Western blot analysis that both start codons may be used in vivo. Interestingly, only expression of the longer version resulted in soluble protein in $E$. coli (our unpublished data). This surprising new regulatory aspect of $s s g A$ requires further analysis.

\section{How do SALPs function?}

Considering that all ssg mutants invariably had defects in peptidoglycan synthesis or autolytic peptidoglycan breakdown, a direct link between the fate of peptidoglycan and SALP functions was proposed (Noens et al. 2005). Detailed microscopic analysis of the respective mutants as well as localization studies resulted in a model for the function of the various SALPs in the control of sporulation of $S$. coelicolor (Noens et al. 2005, Fig. 3). As discussed above, SsgA activates sporulation-specific cell division with perhaps $\mathrm{SsgC}$ as antagonist, $\mathrm{SsgB}$ localises to the growing septa and is important for the cessation of 


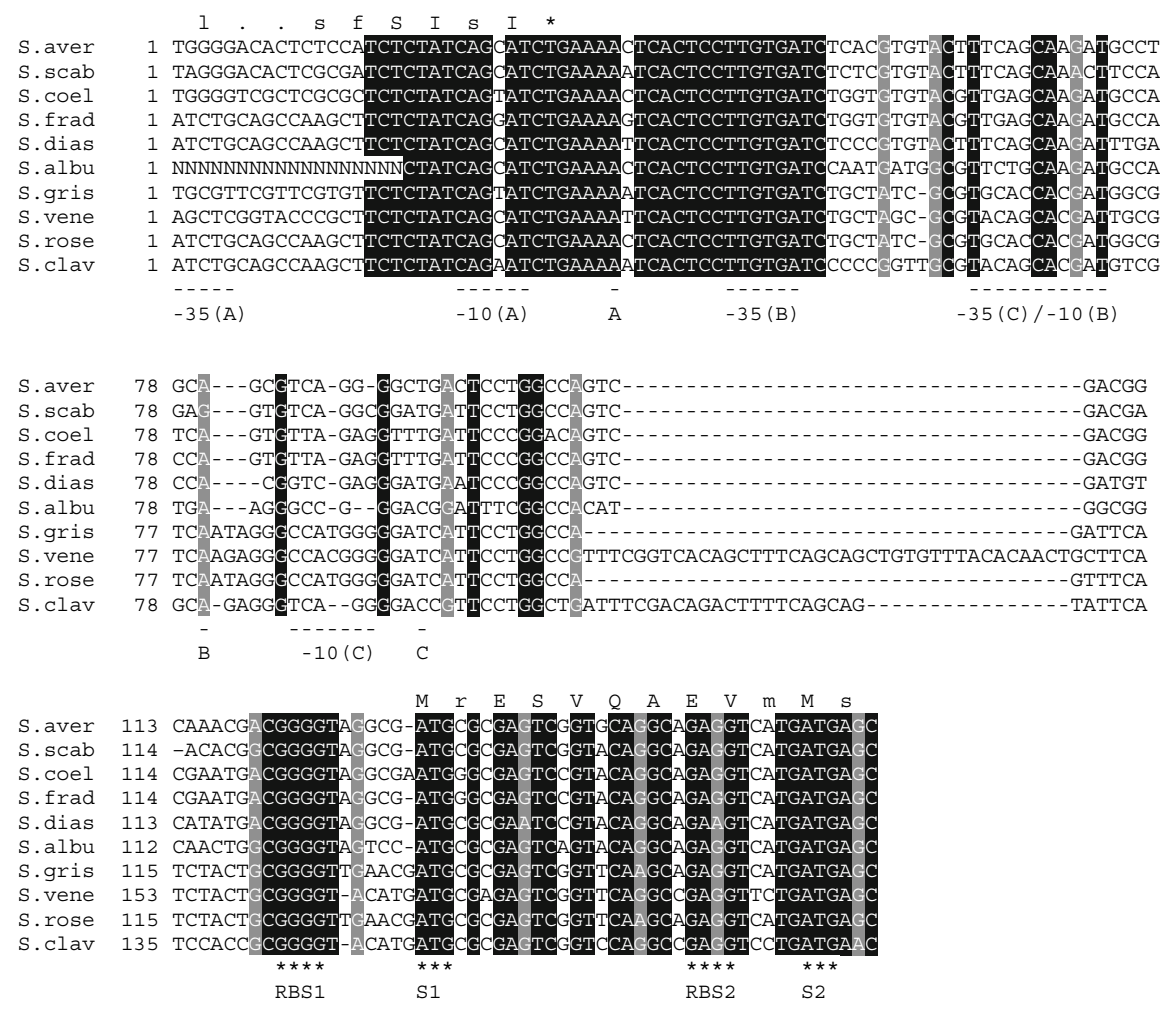

Fig. 2 Alignment of $\operatorname{ssg} A$ promoter regions. Alignment was produced using the Boxshade program (www.ch.embnet.org/ software/BOX_form.html). Only completely conserved nucleotides are shaded; nucleotides shaded in light grey refer to conserved purines. The two alternative start codons (S) for $s s g A$ and their respective ribosome binding sites (RBS) are indicated below the aligned sequence. The two transcriptional start sites and their respective -35 and -10 recognition

aerial tip growth, $s s g D$ mutants have a defective cell wall, SsgE and SsgF ensure the correct autolysis of the peptidoglycan between spores during maturation and SsgG ensures that all sporulation septa are formed at the same time. The SALP protein sequences do not contain any motifs to indicate that they themselves possess enzymatic activity. This suggests that SALPs may control the activity and/or localization of cell-wall enzymes such as PBPs and autolysins. Interestingly, analysis of a functional SsgA-GFP fusion revealed that SsgA localizes dynamically during development and invariably to sites where future restructuring of the peptidoglycan takes place (Noens et al. 2007, Fig. 4). SsgA-GFP localises to germination sites, to the tips of growing aerial hyphae, to septum sites and to branching sites, in line with the idea that SALPs functionally relate to sequences from S. griseus (Yamazaki et al. 2003) and S. coelicolor (Traag et al. 2004) are underlined, where "A" refers to p1 from $S$. griseus, "B" to p1 from $S$. coelicolor or $\mathrm{p} 2$ from S. griseus, and "C" to $\mathrm{p} 2$ from $S$. coelicolor. Consensus amino acid sequences of the C-terminus of SsgR proteins and the $\mathrm{N}$-terminus of SsgA proteins are given above the aligned DNA sequences. The TGA stop codon for $s s g R$ is indicated with an asterisk

the initiation of de novo peptidoglycan synthesis. In young aerial hyphae foci are found at regular intervals and relatively distant spacing, while intense foci are found at the growing tips. At the onset of sporulation these foci fade as new foci appear at alternating sides of the hyphae at positions corresponding to the septum sites. After completion of septum formation spores are produced with two foci, one at either 'pole'. These foci correspond to the future sites where germ tubes emerge; germinating wild-type spores on average produce two germ tubes, and these emerge precisely at the position where SsgA-GFP foci are found. The role of SsgA in the control of germination was further shown by the fact that strains overproducing SsgA regularly have six or seven germ tubes protruding from a single spore, while $s s g$ mutants produce fewer germ tubes. In 

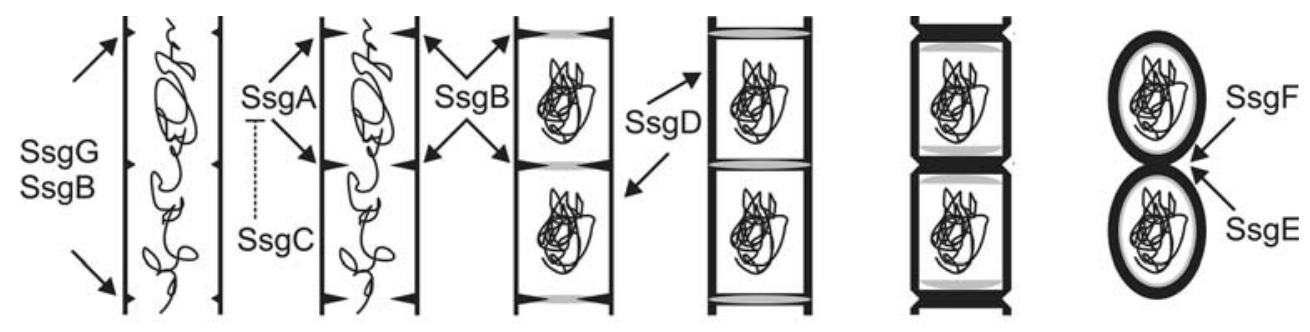

Septal peptidoglycan synthesis

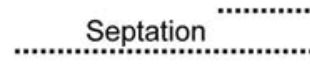

Fig. 3 Model for the proposed functions of the SALPs in the control of morphogenesis of $S$. coelicolor. Arrows indicate the approximate time and place of action for the SALPs, namely septum-site localization (SsgABG), septum growth ( $\mathrm{SsgB}$ ),
Lateral peptidoglycan synthesis

$$
\text { Autolysis }
$$

spore wall synthesis (SsgD) and autolytic spore separation (SsgEF). Considering its opposite effect on cell division, SsgC is proposed to act as an antagonist of SsgA. Adapted from (Noens et al. 2005)
Fig. 4 Dynamic

localization of SsgA-GFP.

Fluorescence micrographs of SsgA-GFP localization during the several stages of development of $S$.

coelcolor. The width of the vegetative hyphae is around $400 \mathrm{~nm}(\mathbf{a}, \mathbf{b})$, that of aerial hyphae and spores is around $800 \mathrm{~nm}(\mathbf{c}-\mathbf{f})$. For further details see (Noens et al. 2007)
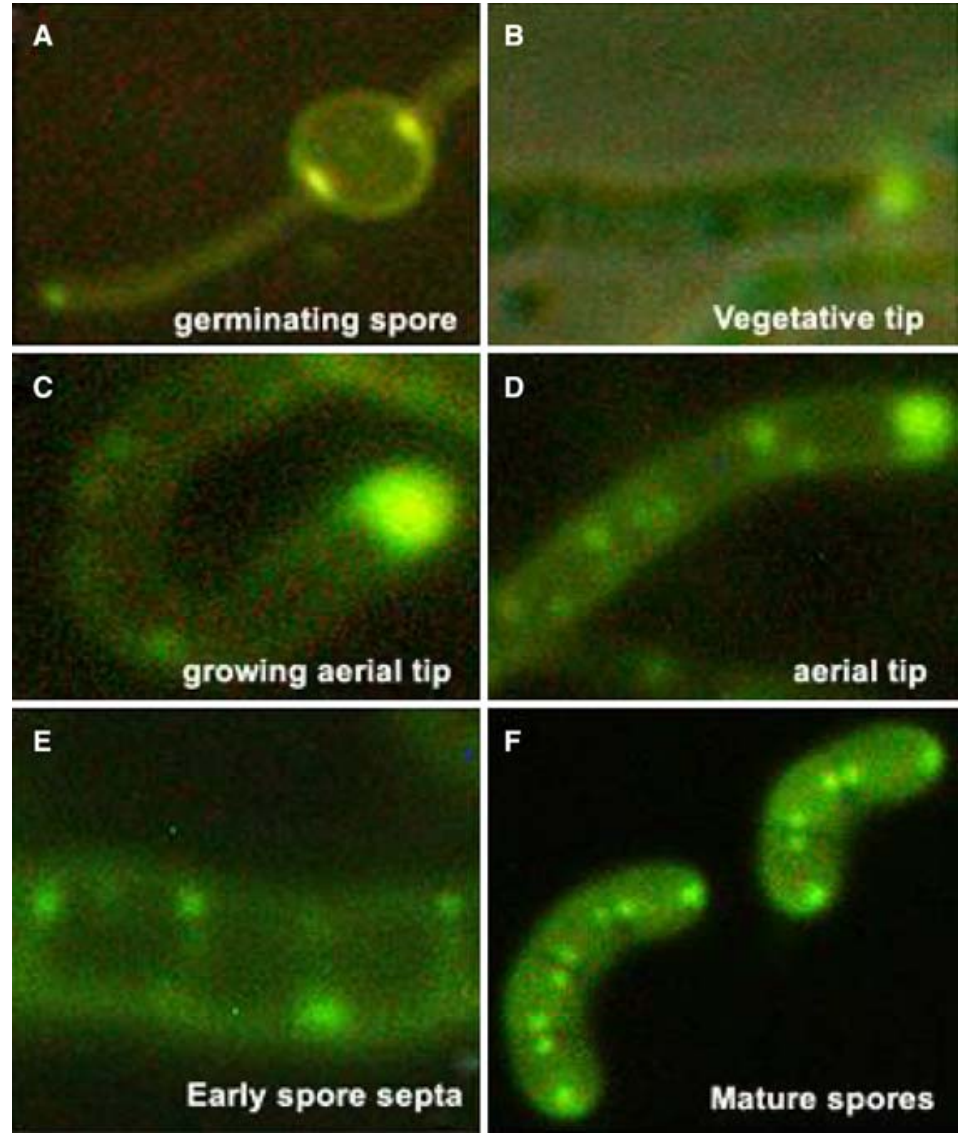

strains expressing SsgA-GFP, signals were lost after several weeks of storage, but even after decades of storage in the freezer wild-type spores still germinate in a highly reproducible fashion, indicating that the germination sites are permanently labelled. This suggests that SsgA does not directly assist in germ tube formation, but rather marks the cell wall to identify future sites. Furthermore, overexpression of 
ssgA in $S$. coelicolor makes the mycelium very sensitive to heat, high sucrose concentrations, and SDS-treatment, all indicating a weakened cell wall. For example, such SsgA-overproducing strains fail to grow in YEME medium (30\% sucrose), We speculate that SsgA, and perhaps all SALPs, functions by recruiting (a) cell wall modifying enzyme(s) to specific locations at the cell wall.

As for how the SALPs themselves are recruited, we have strong evidence that SsgA and SsgB are recruited prior to Z-ring formation, and hence their localization is not dependent on the divisome (Joost Willemse, BT and GPvW, unpublished data). We recently identified mutants in hypothetical ORFs that have an almost identical phenotype as that of SsgG, producing large spores with multiple well-segregated chromosomes, One of the genes encodes a coiled coil protein, and hence may have a cytoskeletal function, tentatively pointing at a functional relationship between $\mathrm{SsgG}$ and the Streptomyces cytoskeleton. Live-cell imaging and biochemical methods such as FRET-FLIM microscopy and two-hybrid screening should elucidate the interaction partners for the SALPs.

\section{Application of SsgA for improved industrial fermentations}

Biotechnological relevance of actinomycetes in general, and streptomycetes in particular, is underlined by the fact that approximately $60 \%$ of all known antibiotics are produced by these organisms, as well as a large number of other biotechnologically interesting compounds and enzymes (Bennett 1998; Demain 1991; Hopwood et al. 1995). Productivity and hence the fermentation costs are strongly affected by the morphology of filamentous microorganisms. Models for mycelial growth have been worked out for filamentous fungi, and particularly for Penicillium chrysogenum (Krabben 1997; Nielsen et al. 1995; Trinchi 1971). Morphology is determined by the efficiency of germination, the tip extension rate, the degree of branching, and especially by fragmentation of the hyphae. When one considers that filamentous microorganisms can produce clumps of millimeters in diameter, the necessity to improve growth by reducing pellet formation becomes obvious. In mycelial pellets the bulk of the biomass is hidden at the inside of the clump, resulting in strongly reduced growth rates and inefficient transfer of nutrients and oxygen. Mycelial mats (large open structures) result in highly viscous broths, which is again undesirable from the production perspective. For streptomycetes the degree of hyphal fragmentation (the major determinant of mycelial clump size) is directly proportional to the frequency of septation (cross-wall formation) of the vegetative hyphae. In turn this directly depends on the $s s g A$ expression level, and as a consequence the morphology of liquid-grown mycelia is dictated by SsgA (van Wezel et al. 2004; van Wezel et al. 2000a). The enhanced expression of SsgA was employed successfully to obtain fragmented and fast growth of pellet-forming species such as S. coelicolor, S. lividans and S. roseosporus in shake flasks as well as in small-scale fermentations (5-50 liter scale) (van Wezel et al. 2006). Using the secreted enzyme tyrosinase as a test enzyme, an increase in the yield of around 3-fold was achieved in significantly shorter fermentation time, underlining the promise of this technology (van Wezel et al. 2006; van Wezel and Vijgenboom 2003).

\section{Conclusions and future perspectives}

SALPs comprise a family of unique proteins, each playing an important role in the control of morphogenesis in streptomycetes. The presence of $\mathrm{SsgB}$ orthologues in morphologically very distinct actinomycetes which can, at least in part, complement each others functions raises a number of important points. First, since SsgB is essential for sporulation in streptomycetes, an important question that needs to be answered is what paralogous function SsgB has in actinomycetes that do not produce aerial hyphae (Salinispora and Thermobifida fusca) or even fail to produce any spores (Acidothermus cellulolyticus, Kineococcus radiotolerans, and Nocardioides sp. JS614). Second, if SALPs, as previously suggested (Noens et al. 2005; Noens et al. 2007) fulfill a recruiting role, the putative $\mathrm{SsgB}$ interacting partner is also likely to be conserved in the genomes of all SALP-containing actinomycetes. The genome sequences of Acidothermus cellulolyticus and Thermobifida fusca can prove very useful on the search for or elimination of such candidate partners, especially because of their considerably smaller sizes (around 2.4 and 3.6 Mbp, respectively), which allows searching for the proverbial needle in a considerably smaller haystack. 
Multiple SALP-encoding genes have so far only been found in actinomycetes that produce aerial hyphae and complex multisporous structures, namely in streptomycetes, in sporangia-forming Frankia species and in Saccharopolyspora erythraea that produces short spore chains. From this one could conclude that SALPs are crucial for septum formation in actinomycetes, that at least two SALPs are required to produce more than one septum simultaneously, and that multiple (three or more) SALPs are required to coordinate the production of long spore chains or sporangia. It would be interesting to see if multiple SALPs can trigger the production of multisporous structures in actinomycetes that normally produce one spore or no spores at all. Conversely, combinations of SALP mutants in for example S. coelicolor may result in streptomycetes producing single spores or short spore chains.

Studies on the three-dimensional structure of SsgA or any other member of the SALP family will hopefully finally identify proteins with significant similarity to the SALPs, and it is to be expected that functional insights may be gleaned from such structural homologies. The overall sequence similarity is highly suggestive of an essential common aspect in their specialized functions. Further functional and localization studies on the SALPs should provide more detailed insight into the exact mode of action of the members of this still rather mysterious protein family.

Acknowledgements We are grateful to Elke Noens and Joost Willemse for sharing unpublished data and for discussions, and to Keith Chater, Barend Kraal and Erik Vijgenboom for discussions. We would like to thank the participants at ISBA14 in August 2007 in Newcastle (UK) for stimulating discussions. This work was supported by grants from the Netherlands Society for Scientific Research (NWO) and from the Royal Netherlands Academy for Arts and Sciences (KNAW) to GPvW.

Open Access This article is distributed under the terms of the Creative Commons Attribution Noncommercial License which permits any noncommercial use, distribution, and reproduction in any medium, provided the original author(s) and source are credited.

\section{References}

Autret S, Errington J (2001) Dynamic proteins in bacteria. Dev Cell 1:10-1

Bennett JW (1998) Mycotechnology: the role of fungi in biotechnology. J Biotechnol 66:101-7
Botta GA, Park JT (1981) Evidence for involvement of penicillin-binding protein 3 in murein synthesis during septation but not during cell elongation. J Bacteriol 145:333-40

Chater KF (1998) Taking a genetic scalpel to the Streptomyces colony

Chater KF, Chandra G (2006) The evolution of development in Streptomyces analysed by genome comparisons. FEMS Microbiol Rev 30:651-672

Chater KF, Losick R (1997) Mycelial life style of Streptomyces coelicolor A3(2) and itrs relatives. In: Dworkin M (eds) Bacteria as multicellular organisms. Oxford University Press, New York, pp 149-182

Claessen D, Rink R, de Jong W, Siebring J, de Vreugd P, Boersma FG, Dijkhuizen L, Wosten HA (2003) A novel class of secreted hydrophobic proteins is involved in aerial hyphae formation in Streptomyces coelicolor by forming amyloid-like fibrils. Genes Dev 17:1714-1726

Claessen D, Stokroos I, Deelstra HJ, Penninga NA, Bormann C, Salas JA, Dijkhuizen L, Wosten HA (2004) The formation of the rodlet layer of streptomycetes is the result of the interplay between rodlins and chaplins. Mol Microbiol 53:433-443

Del Sol R, Mullins JGL, Grantcharova N, Flärdh K, Dyson P (2006) Influence of CrgA on assembly of the cell division protein FtsZ during development of Streptomyces coelicolor. J Bacteriol 188:1540-1550

Demain AL (1991) Production of beta-lactam antibiotics and its regulation. Proc Natl Sci Counc Repub China B 15:251-265

Den Blaauwen T, Aarsman ME, Vischer NO, Nanninga N (2003) Penicillin-binding protein PBP2 of Escherichia coli localizes preferentially in the lateral wall and at midcell in comparison with the old cell pole. Mol Microbiol 47:539-547

Elliot MA, Karoonuthaisiri N, Huang J, Bibb MJ, Cohen SN, Kao CM, Buttner MJ (2003) The chaplins: a family of hydrophobic cell-surface proteins involved in aerial mycelium formation in Streptomyces coelicolor. Genes Dev 17:1727-1740

Errington J, Daniel RA, Scheffers DJ (2003) Cytokinesis in bacteria. Microbiol Mol Biol Rev 67:52-65, table of contents

Flärdh K (2003) Growth polarity and cell division in Streptomyces. Curr Opin Microbiol 6:564-571

Flärdh K, Leibovitz E, Buttner MJ, Chater KF (2000) Generation of a non-sporulating strain of Streptomyces coelicolor A3(2) by the manipulation of a developmentally controlled ftsZ promoter. Mol Microbiol 38:737-749

Flärdh K, van Wezel GP (2003) Cell division during growth and development of Streptomyces. In: Pandalai SG (eds) Recent developments in bacteriology. Transworld research network, Trivandrum, India

Grantcharova N, Lustig U, Flärdh K (2005) Dynamics of FtsZ assembly during sporulation in Streptomyces coelicolor A3(2). J Bacteriol 187:3227-3237

Hao J, Kendrick KE (1998) Visualization of penicillin-binding proteins during sporulation of Streptomyces griseus. J Bacteriol 180:2125-2132

Heidrich C, Ursinus A, Berger J, Schwarz H, Holtje JV (2002) Effects of multiple deletions of murein hydrolases on 
viability, septum cleavage, and sensitivity to large toxic molecules in Escherichia coli. J Bacteriol 184:6093-6099

Holtje JV (1998) Growth of the stress-bearing and shapemaintaining murein sacculus of Escherichia coli. Microbiol Mol Biol Rev 62:181-203

Hopwood DA (1999) Forty years of genetics with Streptomyces: from in vivo through in vitro to in silico. Microbiology 145:2183-2202

Hopwood DA, Chater KF, Bibb MJ (1995) Genetics of antibiotic production in Streptomyces coelicolor A3(2), a model streptomycete. Biotechnology 28:65-102

Horinouchi S, Beppu T (1994) A-factor as a microbial hormone that controls cellular differentiation and secondary metabolism in Streptomyces griseus. Mol Microbiol 12:859-864

Jiang H, Kendrick KE (2000) Characterization of ssfR and ssgA, two genes involved in sporulation of Streptomyces griseus. J Bacteriol 182:5521-5529

Kawamoto S, Ensign JC (1995) Cloning and characterization of a gene involved in regulation of sporulation and cell division in Streptomyces griseus. Actinomycetologica 9:136-151

Kawamoto S, Watanabe H, Hesketh A, Ensign JC, Ochi K (1997) Expression analysis of the ssgA gene product, associated with sporulation and cell division in Streptomyces griseus. Microbiology 143:1077-1086

Keijser BJ, Noens EE, Kraal B, Koerten HK, van Wezel GP (2003) The Streptomyces coelicolor ssgB gene is required for early stages of sporulation. FEMS Microbiol Lett 225:59-67

Kelemen GH, Viollier PH, Tenor J, Marri L, Buttner MJ, Thompson CJ (2001) A connection between stress and development in the multicellular prokaryote Streptomyces coelicolor A3(2). Mol Microbiol 40:804-814

Kormanec J, Sevcikova B (2002) The stress-response sigma factor $\operatorname{sigma}(\mathrm{H})$ controls the expression of $\operatorname{ssgB}$, a homologue of the sporulation-specific cell division gene ssgA, in Streptomyces coelicolor A3(2). Mol Genet Genomics 267:536-543

Krabben P (1997) Morphology of Penicillium chrysogenum. Thesis. Type, Technical University of Denmark, Lyngby, Denmark

Lee EJ, Cho YH, Kim HS, Roe JH (2004) Identification of sigmaB-dependent promoters using consensus-directed search of Streptomyces coelicolor genome. J Microbiol 42:147-151

Lowe J, van den Ent F, Amos LA (2004) Molecules of the bacterial cytoskeleton. Annu Rev Biophys Biomol Struct 33:177-198

Marston AL, Thomaides HB, Edwards DH, Sharpe ME, Errington J (1998) Polar localization of the MinD protein of Bacillus subtilis and its role in selection of the mid-cell division site. Genes Dev 12:3419-3430

Mazza P, Noens EE, Schirner K, Grantcharova N, Mommaas AM, Koerten HK, Muth G, Flärdh K, van Wezel GP, Wohlleben W (2006) MreB of Streptomyces coelicolor is not essential for vegetative growth but is required for the integrity of aerial hyphae and spores. Mol Microbiol 60:838-852

McCormick JR, Su EP, Driks A, Losick R (1994) Growth and viability of Streptomyces coelicolor mutant for the cell division gene ftsZ. Mol Microbiol 14:243-254
Nielsen J, Johansen CL, Jacobsen M, Krabben P, Villadsen J (1995) Pellet formation and fragmentation in submerged cultures of Penicillium chrysogenum and its relation to penicillin production. Biotechnol Prog 11:93-98

Noens EE, Mersinias V, Traag BA, Smith CP, Koerten HK, van Wezel GP (2005) SsgA-like proteins determine the fate of peptidoglycan during sporulation of Streptomyces coelicolor. Mol Microbiol 58:929-944

Noens EE, Mersinias V, Willemse J, Traag BA, Laing E, Chater KF, Smith CP, Koerten HK, van Wezel GP (2007) Loss of the controlled localization of growth stage-specific cell-wall synthesis pleiotropically affects developmental gene expression in an ssgA mutant of Streptomyces coelicolor. Mol Microbiol 64:1244-1259

Ohnishi Y, Yamazaki H, Kato JY, Tomono A, Horinouchi S (2005) AdpA, a central transcriptional regulator in the Afactor regulatory cascade that leads to morphological development and secondary metabolism in Streptomyces griseus. Biosci Biotechnol Biochem 69:431-439

Schwedock J, Mccormick JR, Angert ER, Nodwell JR, Losick R (1997) Assembly of the cell division protein ftsz into ladder like structures in the aerial hyphae of Streptomyces coelicolor. Mol Microbiol 25:858

Sevcikova B, Benada O, Kofronova O, Kormanec J (2001) Stress-response sigma factor sigma(H) is essential for morphological differentiation of Streptomyces coelicolor A3(2). Arch Microbiol 177:98-106

Stewart GC (2005) Taking shape: control of bacterial cell wall biosynthesis. Mol Microbiol 57:1177-1181

Thompson JD, Gibson TJ, Plewniak F, Jeanmougin F, Higgins DG (1997) The CLUSTAL_X windows interface: flexible strategies for multiple sequence alignment aided by quality analysis tools. Nucleic Acids Res 25:4876-4882

Traag BA, Kelemen GH, Van Wezel GP (2004) Transcription of the sporulation gene ssgA is activated by the IclR-type regulator SsgR in a whi-independent manner in Streptomyces coelicolor A3(2). Mol Microbiol 53:985-1000

Traag BA, Seghezzi N, Vijgenboom E, van Wezel GP (2007) Characterization of the sporulation control protein SsgA by use of an efficient method to create and screen random mutant libraries in streptomycetes. Appl Environ Microbiol 73:2085-2092

Trinchi APJ (1971) A study of kinetics of hyphal extension and branch initiation of fungal mycelia. J Gen Microbiol 81:225-236

van Wezel GP, van der Meulen J, Kawamoto S, Luiten RGM, Koerten HK, Kraal B (2000a) ssgA is essential for sporulation of Streptomyces coelicolor A3(2) and affects hyphal development by stimulating septum formation. J Bacteriol 182:5653-5662

van Wezel GP, van der Meulen J, Taal E, Koerten H, Kraal B (2000b) Effects of increased and deregulated expression of cell division genes on the morphology and on antibiotic production of streptomycetes. Antonie Van Leeuwenhoek 78:269-276

van Wezel GP, Vijgenboom E (2003) Improved growth characteristics of filamentous microorganisms. Patent Application WO 2004/041858 A1

van Wezel GP, Luiten RG, Kraal B (2004) Reducing branching and enhancing fragmentation of culturing filamentous microorganisms. US patent 09/749,185 
van Wezel GP, Krabben P, Traag BA, Keijser BJ, Kerste R, Vijgenboom E, Heijnen JJ, Kraal B (2006) Unlocking Streptomyces spp. for use as sustainable industrial production platforms by morphological engineering. Appl Environ Microbiol 72:5283-5288

Wildermuth H, Hopwood DA (1970) Septation during sporulation in Streptomyces coelicolor. J Gen Microbiol 60:51-59
Wu LJ, Errington J (2004) Coordination of cell division and chromosome segregation by a nucleoid occlusion protein in Bacillus subtilis. Cell 117:915-925

Yamazaki H, Ohnishi Y, Horinouchi S (2003) Transcriptional switch on of ssgA by A-factor, which is essential for spore septum formation in Streptomyces griseus. J Bacteriol 185:1273-1283 